\title{
ON TOPOLOGICAL PROPERTIES OF SETS ADMITTING VARISOLVENT FUNCTIONS
}

\author{
D. BRAESS
}

AbStract. Mairhuber's theorem on Haar subspaces is generalized for the nonlinear case, where varisolvent functions are considered.

1. According to a well-known theorem of Mairhuber [4] a compact set in $\boldsymbol{R}^{N}$ is homeomorphic to a subset of a circumference $\Gamma$, if it admits a real Haar subspace with dimension $N \geqq 2$. This result has been proved for general compact spaces by Curtis [2] and by Sieklucki [7]. A first extension of Mairhuber's theorem to nonlinear families of functions was given by Dunham [3]. In this note we will derive a stronger result.

THEOREM 1. Let the compact set $Q$ admit a varisolvent family of functions. If the degree of solvence is bounded and if the maximal degree is greater than 1, then $Q$ is homeomorphic to a subset of a circumference $\Gamma$. Moreover, if the degree is an even number at some element, then the subset must be proper.

As a consequence of this theorem it is natural to consider varisolvent functions only on intervals in $\boldsymbol{R}$. This is the specialization used throughout the literature. The principal part of the proof will treat the case where the degree $n$ is 2. Afterwards, the induction for $n>2$ proceeds as in the proofs for linear families presented in [6], [8, p. 218].

2. We define varisolvent functions on a compact set $Q$. Let the real function $F(a, x)$ be defined for $x \in Q$ and $a \in P$, where $P$ is the parameter space [5]. For all $a \in P$ we assume $F(a, \cdot) \in C(Q)$, but there is no need to endow $P$ with a topology.

Definition. (i) $F$ has Property $\mathrm{Z}$ of degree $m$ at $a^{*} \in P$, if for any $a \neq a^{*}, F(a, x)-F\left(a^{*}, x\right)$ has at most $m-1$ zeros for $x \in Q$.

(ii) $F$ is solvent of degree $m$ at $a^{*} \in P$ if given a set of $m$ distinct points $x_{j} \in Q, j=1,2, \cdots, m$, and $\varepsilon>0$, then there exists a

$$
\delta=\delta\left(a^{*}, \varepsilon, x_{1}, x_{2}, \cdots, x_{m}\right)
$$

Received by the editors October 1,1971 .

AMS 1970 subject classifications. Primary 41A65; Secondary 41A50.

Key words and phrases. Nonlinear approximation, Mairhuber theorem on Haar subspaces, varisolvency.

(c) American Mathematical Society 1972 
such that $\left|F\left(a^{*}, x_{j}\right)-y_{j}\right|<\delta$ implies the existence of a parameter $a \in P$, satisfying

$$
F\left(a, x_{j}\right)=y_{j}, \quad j=1,2, \cdots, m,
$$

as well as $\left\|F(a, \cdot)-F\left(a^{*}, \cdot\right)\right\|<\varepsilon$.

(iii) $F$ is varisolvent if $F$ possesses both the properties (i) and (ii) with the same degree for each $a \in P$.

At first we prove

LEMMA 2. Let $\Gamma$ be a circumference and $q$ a point outside $\Gamma$. If $F(a, x)$ is varisolvent on $\Gamma$ (resp., on $\Gamma \cup\{q\}$ ), then the degree of solvence is odd (resp., at most 1) at each $a \in P$.

Proof. Let $m=m\left(a^{*}\right)$ be even. Select $m$ distinct points $x_{i} \in \Gamma$ in cyclic order. For sufficiently small $\delta>0$ there exists a solution of

$$
F\left(a, x_{i}\right)=y_{i}, \quad i=1,2, \cdots, m,
$$

where $y_{i}=F\left(a^{*}, x_{i}\right)+(-1)^{i} \delta$. Obviously, $F(a, x)-F\left(a^{*}, x\right)$ has $m$ zeros on $\Gamma$, contradicting Property $Z$. This completes the proof for the statement on $\Gamma$. Concerning $\Gamma \cup\{q\}$, the statement for even $m$ is a consequence of the preceding one. It is sufficient to consider odd degrees $m=m\left(a^{*}\right)$. Here, select $m-1$ distinct points $x_{i} \in \Gamma$ in cyclic order. Define $y_{i}, i=$ $1,2, \cdots, m-1$, as above and set $y_{0}=F\left(a^{*}, q\right)$. Then the solution of

$$
\begin{aligned}
& F(a, x)=y_{i}, \quad i=1,2, \cdots, m-1, \\
& F(a, q)=y_{0}
\end{aligned}
$$

yields a contradiction to Property $\mathrm{Z}$.

3. Before proving the main theorem we will recall the linear case. Let $u_{1}, u_{2}$ be a base of a two dimensional Haar subspace. Then

$$
x \rightarrow\left\{u_{1}(x), u_{2}(x)\right\}
$$

defines an injective continuous mapping of $Q$ into the real projective line. This mapping was considered in [8, p. 221]. If $u_{2}(x)$ has no zero in $Q$, we may use the mapping $\varphi: Q \rightarrow \boldsymbol{R}$ which sends $x$ to $u_{1}(x) / u_{2}(x)$. We may even abandon the assumption on $u_{2}(x)$. By virtue of a lemma of Schoenberg and Yang (to be mentioned below) it is only necessary to present a mapping of $Q \backslash U$ into $\Gamma$ for each open set $U \subset Q$. For given $U$ choose a function $u_{2}$ without zero in $Q \backslash U$. Moreover, $\varphi$ may be defined by the relation $\varphi(x) \cdot u_{2}(x)=u_{1}(x)$. This concept will be translated to the nonlinear case.

4. Proof of Theorem 1. Let $F$ be a varisolvent function on $Q$. We shall proceed by induction on $n$, the number of maximal degree of solvence. Choose $a^{*} \in P$ with $m\left(a^{*}\right)=n$. Observe that the translation 
$\tilde{F}(a, x)=F(a, x)-F\left(a^{*}, x\right)$ generates a function $\tilde{F}$, which has the same degrees of Property $\mathrm{Z}$ and solvence as $F$ and which is also varisolvent. Thus we may assume $F\left(a^{*}, x\right) \equiv 0$ without loss of generality.

At first let $n=2$ and let $U$ be an arbitrary nonvoid open set in $Q$. Select $q_{1} \in U$ and $q_{2} \in M:=Q \backslash U$. From the definition of solvence we know that there exists a $\delta>0$ and a mapping from the unit square in $\boldsymbol{R}^{2}$ into $C(Q)$ :

$$
\left(y_{1}, y_{2}\right) \rightarrow F\left(a\left(y_{1}, y_{2}\right), \cdot\right) \text {, }
$$

such that

$$
F\left(a\left(y_{1}, y_{2}\right), q_{i}\right)=\delta \cdot y_{i}, \quad i=1,2 .
$$

Since $m\left(a^{*}\right)$ is maximal, it follows from Property $\mathrm{Z}$ that the mapping is uniquely defined for fixed $\delta$. By virtue of Theorem 1 in [1] the mapping is continuous.

For $-1 \leqq t \leqq+1$ set $a_{t}=a(0, t)$. Obviously, we have $F\left(a_{0}, \cdot\right)=F\left(a^{*}, \cdot\right)$ and $F\left(a_{t}, q_{1}\right)=0$. Hence, $m\left(a_{t}\right)=2$ and Property $\mathrm{Z}$ implies

$$
F\left(a_{t}, x\right) \neq F\left(a_{s}, x\right) \neq 0, \quad x \in M,
$$

for all $s, t \in[-1,+1], s \neq t \neq 0$. Since $M$ is compact, it follows that

$$
\min _{x \in M} \min \left\{\left|F\left(a_{1}, x\right)\right|,\left|F\left(a_{-1}, x\right)\right|\right\}=\eta>0 .
$$

Furthermore, from the solvence property at $a^{*}$ we conclude that there exists a parameter $b \in P$ such that

$$
F\left(b, q_{1}\right) \neq 0
$$

and

$$
\|F(b, \cdot)\|<\eta \text {. }
$$

Now we are ready to define a mapping $\varphi: M \rightarrow[-1,+1]$ by

$$
F\left(a_{\varphi(x)}, x\right)=F(b, x), \quad x \in M .
$$

We claim that the mapping is well defined. Indeed, by virtue of (4), (5) and (6) we have

or

$$
F\left(a_{1}, x\right) \geqq \eta \quad \text { and } \quad F\left(a_{-1}, x\right) \leqq-\eta,
$$

$$
F\left(a_{-1}, x\right) \geqq \eta \quad \text { and } \quad F\left(a_{1}, x\right) \leqq-\eta,
$$

for each $x \in M$. Hence, for each $x \in M$ the relation $F\left(a_{t}, x\right)=F(b, x)$ holds for at least one $t \in[-1,+1]$. By virtue of (5), this value is unique. Furthermore, $\varphi$ is injective. If $\varphi\left(x_{1}\right)=\varphi\left(x_{2}\right)=t$, then $F\left(a_{t}, \cdot\right)-F(b, \cdot)$ has two zeros, which implies $F\left(a_{t}, \cdot\right) \equiv F(b, \cdot)$, contradicting $F\left(b, q_{1}\right) \neq$ $F\left(a_{t}, q_{1}\right)=0$. Finally, from the continuity of the mapping $[-1,+1] \times$ $M \ni(t, x) \rightarrow F\left(a_{t}, x\right)-F(b, x)$, from compactness, and from the injectivity 
of $\varphi$, we conclude that $\varphi$ is continuous. Hence, $M=Q \backslash U$ is homeomorphic to a subset of $\boldsymbol{R}$ or a subset of $\Gamma$. Since this holds for each open set $U$, from Lemma 1 in [6] (the same as Lemma 2.4 in [8, p. 219]), we obtain that $Q$ can be homeomorphically embedded into $\Gamma$, or $Q$ is homeomorphic to a union of $\Gamma$ and a single point $q \notin \Gamma$. The second case is excluded by virtue of Lemma 2. Thus, the statement for $n=2$ is established.

Now, let $n>2$. Assume that Theorem 1 is true for $n-1$. Let $U$ be an arbitrary open set in $Q$. Choose $q \in U$ and $a^{*} \in P$ with $m\left(a^{*}\right)=n$. Set

$$
P^{\prime}=\left\{a \in P: F(a, q)=F\left(a^{*}, q\right)\right\} .
$$

Obviously, $F(a, x)$ is varisolvent for $x \in Q \backslash U$ and $a \in P^{\prime}$. By this restriction the degree of solvence is reduced by one for each $a \in P^{\prime}$. Since $a^{*} \in P^{\prime}$, the induction hypothesis assures that $Q \backslash U$ is homeomorphic to a subset of $\Gamma$.

By the same arguments as those used for the case $n=2$, the proof of the theorem is completed.

\section{REFERENCES}

1. D. Braess, On varisolvency and alternation, J. Approximation Theory (to appear).

2. P. C. Curtis, Jr., n-parameter families and best approximation, Pacific J. Math. 9 (1959), 1013-1027. MR 21 \#7385.

3. C. B. Dunham, Unisolvence on multidimensional spaces, Canad. Math. Bull. 11 (1968), 469-474. MR 38 \#3671.

4. J. C. Mairhuber, On Haar's theorem concerning Chebychev approximation problems having unique solutions, Proc. Amer. Math. Soc. 7 (1956), 609-615. MR 18, 125.

5. J. R. Rice, The approximation of functions, Vol. 2: Nonlinear and multivariate theory, Addison-Wesley, Reading, Mass., 1969. MR 39 \#5989.

6. I. J. Schoenberg and C. T. Yang, On the unicity of solutions of problems of best approximation, Ann. Mat. Pura Appl. (4) 54 (1961), 1-12. MR 25 \#5324.

7. K. Sieklucki, Topological properties of sets admitting the Tschebycheff systems, Bull. Acad. Polon. Sci. Sér. Sci. Math. Astronom. Phys. 6 (1958), 603-606. MR 20 \#6625.

8. I. Singer, Best approximation in normed vector spaces by elements of vector subspaces, Editura Academiei Republicii Socialiste România, Bucharest, 1967; English transl., Die Grundlehren der math. Wissenschaften, Band 171, Springer-Verlag, New York and Berlin, 1970. MR 38 \#3677; MR 42 \#4937.

INSTITUT FÜR NUmerische UND InStRUmentelle Mathematik, WestFalische Wilhelms-Universität, 44 Münster, Federal Republic of Germany 